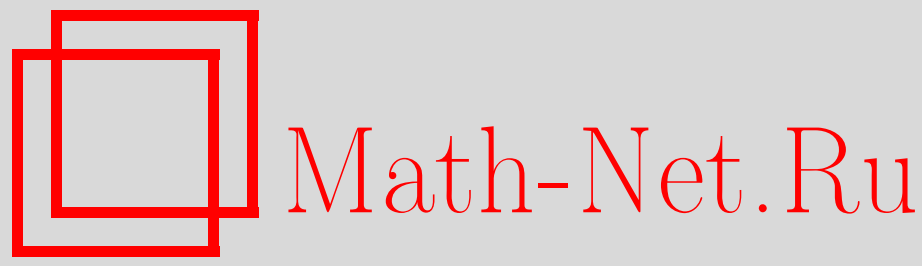

О. В. Ильин, Стационарные решения кинетической модели Бродуэлла, ТМФ, 2012, том 170, номер 3, 481-488

DOI: https://doi.org/10.4213/tmf6780

Использование Общероссийского математического портала Math-Net.Ru подразумевает, что вы прочитали и согласны с пользовательским соглашением http: //www . mathnet.ru/rus/agreement

Параметры загрузки:

IP: 54.80 .73 .141

26 апреля 2023 г., $15: 33: 46$

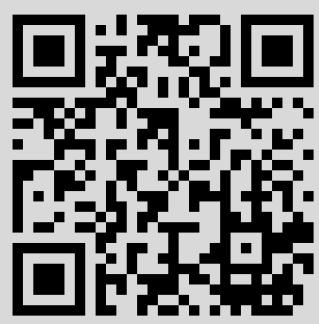




\title{
СТАЦИОНАРНЫЕ РЕШЕНИЯ КИНЕТИЧЕСКОЙ МОДЕЛИ БРОДУЭЛЛА
}

\begin{abstract}
Рассмотрена стационарная дискретная модель уравнения Больцмана для четырех скоростей (модель Бродуэлла). Для модели получены новые точные автомодельные решения, отвечающие несжимаемому и сжимаемому газу. Показано, что один из классов решений удовлетворяет задаче об испарении и конденсации газа на границе круга и внешнего пространства. Оказывается, что система сильно неравновесна и для нее неприменимы уравнения сплошной среды.
\end{abstract}

Ключевые слова: кинетическая модель Бродуэлла, автомодельные решения.

\section{1. ВВЕДЕНИЕ}

В силу высокой сложности исследования уравнения Больцмана (УБ) часто прибегают к рассмотрению дискретных моделей УБ [1]. Для таких моделей число возможных скоростей движений частиц ограничено и вместо интегро-дифференциального УБ дискретные модели представляются в виде систем нелинейных уравнений в частных производных.

Первый систематический поиск решений для дискретных моделей УБ с числом скоростей от четырех и более проведен в работах [2], [3]. Эти решения нестационарны и имеют вид бегущих волн. Также для построения стационарных и нестационарных решений четырехскоростной модели (система Бродуэлла на плоскости) использовался метод потенииальных течений [4], [5]. Еще один метод основан на отображении решений двухскоростной модели Карлемана в решения стационарной модели Бродуэлла [6], [7]. Частные решения также могут быть найдены с помощью усеченных рядов Пенлеве [8].

В нестационарном случае наблюдаются эффекты, не имеющие физического смысла. Например, существуют решения в виде волн, движущихся со скоростью, большей, чем скорость частиц [9]. В стационарном случае многие физически интересные задачи (распространение газа между двумя полубесконечными пластинами, истечение газа из полости [9]) описываются качественно верно моделью Бродуэлла. Также имеется еще одна причина обратиться к исследованию стационарного случая. В работе [10] было численно найдено, что в окрестности стационарных решений простейшей дискретной модели - системы Карлемана - обнаруживается переход к хаосу по

* Вычислительный центр им. А. А. Дородницына РАН, Москва, Россия. E-mail: oilyin@gmail.com 
сценарию Фейгенбаума (через последовательность удвоений периода), т. е. кинетические уравнения при определенных условиях могут рассматриваться как модели пространственно неоднородной турбулентности. Более того, численные расчеты для УБ показывают, что для некоторых краевых задач при уменьшении числа Кнудсена наблюдаются хаотические режимы [11].

Интересно рассмотреть физически более содержательные дискретные модели УБ, чем система Карлемана. Все дискретные модели УБ допускают решения в автомодельном виде. Этот класс решений остается малоисследованным. Единственный нетривиальный результат был получен для трехскоростной модели в нестационарном случае [12].

Рассмотрим стационарную четырехскоростную дискретную модель УБ на плоскости (модель Бродуэлла) в стандартной безразмерной форме [13]:

$$
\frac{\partial f_{1}}{\partial x}=-\frac{\partial f_{2}}{\partial x}=-\frac{\partial f_{3}}{\partial y}=\frac{\partial f_{4}}{\partial y}=I(f), \quad I(f) \equiv f_{3} f_{4}-f_{1} f_{2},
$$

где $f_{j}(x, y), j=1, \ldots, 4,-$ плотности частиц, движущихся на плоскости в направлениях $\pm x, \pm y$ с единичной по модулю скоростью. Найдем автомодельные решения системы (1) и приведем пример краевой задачи, к которой можно применить найденные решения. Естественно, кроме неотрицательности плотностей $f_{j}(x, y)$, $j=1, \ldots, 4$, требуется, чтобы решения допускали диссипацию, т. е. $I(f) \neq 0$.

\section{2. НЕСЖИМАЕМЫЙ ГАЗ}

Основное наблюдение заключается в том, что можно искать решения системы (1) в следующем виде:

$$
\begin{array}{ll}
f_{1}(x, y)=K+\frac{g(\xi)}{y}, & f_{2}(x, y)=K-\frac{g(\xi)}{y}, \\
f_{3}(x, y)=K+\frac{g(\xi)}{x}+\frac{B}{x}, & f_{4}(x, y)=K-\frac{g(\xi)}{x}-\frac{B}{x}
\end{array}
$$

где $B, K$ - некоторые константы, $\xi \equiv y / x$, а $g(\xi)$ - неизвестная функция. В данном случае полная плотность газа постоянна:

$$
\rho(x, y)=\sum_{j=1}^{4} f_{j}(x, y)=4 K
$$

Подставим соотношения (2) в уравнения (1), получаем одно уравнение типа Риккати для функции $g(\xi)$ :

$$
g_{\xi}=\left(1-\xi^{-2}\right) g^{2}+2 B g+B^{2} .
$$

Рассмотрим частные случаи уравнения (3).

Случай А: Пусть $B=0$. В уравнении (3) разделяются переменные, и оно легко интегрируется:

$$
g(\xi)=-\frac{\xi}{\xi^{2}+C \xi+1}
$$


где $C$ - постоянная интегрирования. Тогда решения модели Бродуэлла выглядят следующим образом:

$$
\begin{aligned}
& f_{1,2}(x, y)=K \mp \frac{x}{y^{2}+C x y+x^{2}}, \\
& f_{3,4}(x, y)=K \mp \frac{y}{y^{2}+C x y+x^{2}} .
\end{aligned}
$$

Так как только неотрицательные решения имеют физический смысл, то следует выделить области на плоскости, где правые части равенств (4) неотрицательны. Знаменатель в правых частях (4) приводится к следующему виду:

$$
y^{2}+C x y+x^{2}=\left(y+\frac{C x}{2}\right)^{2}+\left(1-\frac{C^{2}}{4}\right) x^{2} .
$$

Будем рассматривать $|C|<2$. В этом случае решения (4) обращаются в бесконечность только при $x=y=0$, а вне начала координат решения ограничены. Тогда, выбирая любое $R>0$, можно найти такое $K=K(R)>0$, что решения (4) неотрицательны в области $x^{2}+y^{2} \geqslant R$.

Случай Б: Пусть $B \neq 0$. Сделаем следующую замену зависимой переменной в уравнении $(3): g(\xi)=B /(u(\xi)-1)$. Тогда вместо уравнения (3) относительно переменной $u(\xi)$ получаем уравнение Риккати в каноническом виде

$$
B^{-1} u_{\xi}=-u^{2}+\xi^{-2}
$$

Известно, что такое уравнение Риккати имеет общее решение, представимое в элементарных функциях. Проведем линеаризацию уравнения, сделав замену $u(\xi)=$ $B^{-1} v(\xi)^{-1} v(\xi)_{\xi}$, и для функции $v(\xi)$ получим

$$
\xi^{2} v_{\xi \xi}-B^{2} v=0
$$

Общее решение последнего уравнения имеет вид

$$
v(\xi)=C_{1} \xi^{\left(1+\sqrt{1+4 B^{2}}\right) / 2}+C_{2} \xi^{\left(1-\sqrt{1+4 B^{2}}\right) / 2},
$$

где $C_{1}, C_{2}$ - постоянные интегрирования. Возвращаясь к исходной функции $g(\xi)$, получим решения уравнений (1). Для двух частных случаев $C_{1} \neq 0, C_{2}=0$ и $C_{1}=0$, $C_{2} \neq 0$ эти решения имеют особенно простой вид. Например, в случае $C_{1} \neq 0, C_{2}=0$ имеем

$$
\begin{aligned}
& f_{1,2}(x, y)=K \pm \frac{2 B^{2}}{\left(1+\sqrt{1+4 B^{2}}\right) x-2 B y}, \\
& f_{3,4}(x, y)=K \pm \frac{2 B^{2} y}{\left(1+\sqrt{1+4 B^{2}}\right) x^{2}-2 B x y} \pm \frac{B}{x}
\end{aligned}
$$

В случае $C_{1}=0, C_{2} \neq 0$ получаем следующие решения:

$$
\begin{aligned}
& f_{1,2}(x, y)=K \pm \frac{2 B^{2}}{\left(1-\sqrt{1+4 B^{2}}\right) x-2 B y} \\
& f_{3,4}(x, y)=K \pm \frac{2 B^{2} y}{\left(1-\sqrt{1+4 B^{2}}\right) x^{2}-2 B x y} \pm \frac{B}{x}
\end{aligned}
$$


Очевидно, что решения (5) становятся неограниченными на прямых

$$
x=0, \quad\left(1+\sqrt{1+4 B^{2}}\right) x-2 B y=0 .
$$

В областях, не содержащих данных прямых, всегда можно обеспечить неотрицательность решений (5) выбором постоянной $K$. Решения (6) обращаются в бесконечность на прямых

$$
x=0, \quad\left(1-\sqrt{1+4 B^{2}}\right) x-2 B y=0,
$$

и аналогично случаю $C_{1} \neq 0, C_{2}=0$ получаем, что в областях, не содержащих прямых, положительность решений гарантируется выбором $K$.

\section{3. СЖКИМАЕМЫЙ ГАЗ}

Построим решение модели (1) в следующем виде:

$$
\begin{array}{ll}
f_{1}(x, y)=\frac{g(\xi)}{y}+\frac{A}{y}, & f_{2}(x, y)=-\frac{g(\xi)}{y}, \\
f_{3}(x, y)=\frac{g(\xi)}{x}+\frac{A}{x}, & f_{4}(x, y)=-\frac{g(\xi)}{x},
\end{array}
$$

где $A$ - некоторая константа, $\xi \equiv y / x$. Положим $A \neq 0$. Тогда полная плотность газа непостоянна:

$$
\rho(x, y)=\sum_{j=1}^{4} f_{j}(x, y)=\frac{A}{x}+\frac{A}{y} .
$$

Модель Бродуэлла допускает более общий вид решений для сжимаемого газа, содержащий решения вида (7) как частный случай, однако такие решения выражаются квадратурами и рассматриваться не будут. Подставляя равенства (7) в систему (1), получаем одно обыкновенное дифференциальное уравнение относительно функции $g(\xi)$ :

$$
g_{\xi}=\left(1-\xi^{-2}\right) g^{2}+A\left(1-\xi^{-2}\right) g .
$$

Последнее уравнение имеет следующее общее решение в переменных $x, y$ :

$$
g(x, y)=\frac{A}{C e^{-A\left(x^{2}+y^{2}\right) / x y}-1} .
$$

Используя соотношения (7), получаем решения модели Бродуэлла (1):

$$
\begin{aligned}
& f_{1}(x, y)=\frac{A}{y} \cdot \frac{C e^{-A\left(x^{2}+y^{2}\right) / x y}}{C e^{-A\left(x^{2}+y^{2}\right) / x y}-1}, \\
& f_{2}(x, y)=-\frac{A}{y} \cdot \frac{1}{C e^{-A\left(x^{2}+y^{2}\right) / x y}-1}, \\
& f_{3}(x, y)=\frac{A}{x} \cdot \frac{C e^{-A\left(x^{2}+y^{2}\right) / x y}}{C e^{-A\left(x^{2}+y^{2}\right) / x y}-1}, \\
& f_{4}(x, y)=-\frac{A}{x} \cdot \frac{1}{C e^{-A\left(x^{2}+y^{2}\right) / x y}-1} .
\end{aligned}
$$

Найдем области на плоскости, где решения (8) неотрицательны. Будем рассматривать область $x>0, y>0$. Тогда для положительности плотности $\rho=\sum_{j=1}^{4} f_{j}$ необходимо потребовать, чтобы выполнялось условие $A>0$. Теперь, выбирая $C<0$, получаем, что решения (8) положительны в области $x>0, y>0$. 


\section{4. ЗАДАЧИ ОБ ИСПАРЕНИИ И КОНДЕНСАЦИИ ГАЗА}

Рассмотрим модель Бродуэлла (1) в ограниченной прямоугольной области $x \in$ $\left[a_{1}, a_{2}\right], y \in\left[b_{1}, b_{2}\right]$. На границах области ставятся следующие условия (в безразмерных координатах):

$$
\begin{array}{ll}
f_{1}\left(a_{1}, y\right)=F_{1}(y), & f_{2}\left(a_{2}, y\right)=F_{2}(y), \\
f_{3}\left(x, b_{1}\right)=F_{3}(x), & f_{4}\left(x, b_{2}\right)=F_{4}(x),
\end{array}
$$

где $F_{1}(y), F_{2}(y), F_{3}(x), F_{4}(x)$ - плотности частиц на границах. В работе [13] показано, что данная задача разрешима в прямоугольнике, если граничные плотности $F_{j}, j=1, \ldots, 4$, суть непрерывные функции. С физической точки зрения условия (9), (10) определяют испарение газа с границ прямоугольной области. Первое условие в (9) задает плотность частиц, испаряющихся с левой вертикальной границы с единичной скоростью в направлении оси $x$, второе условие в (9) задает плотность частиц, испаряющихся с правой вертикальной границы с единичной скоростью в направлении, противоположном оси $x$. Аналогично трактуются условия (10). Очевидно, что все три класса решений, полученных в настоящей работе, в областях неотрицательности являются решениями задачи (9), (10) для некоторых частных граничных плотностей $F_{j}, j=1, \ldots, 4$.

В работах [6], [9] рассматривалась стационарная система Бродуэлла в полубесконечном канале, в котором продольные стенки считались зеркально отражающими, а на поперечной стенке ставились смешанные граничные условия: часть стенки отражает частицы зеркально-диффузно, другая часть испаряет газ в канал. В настоящей работе мы решаем задачу об испарении и конденсации газа на границе круглой полости на плоскости и внешнего пространства. Рассмотрим плоский дискретный газ, подчиняющийся уравнениям Бродуэлла (1). Пусть в начале координат имеется круг единичного радиуса, граница круга испаряет и конденсирует газ. Далее удобно использовать полярные координаты $(r, \varphi)$. Зададим испарение и конденсацию газа граничными условиями (в безразмерных координатах):

$$
\begin{array}{ll}
\left.f_{1}(r, \varphi)\right|_{r=1}=1-\cos \varphi, & \left.f_{2}(r, \varphi)\right|_{r=1}=1+\cos \varphi, \\
\left.f_{3}(r, \varphi)\right|_{r=1}=1-\sin \varphi, & \left.f_{4}(r, \varphi)\right|_{r=1}=1+\sin \varphi .
\end{array}
$$

Теперь следует поставить условия на бесконечности. Естественно предположить, что распределение газа вдали от круглой полости будет равновесным абсолютно максвелловским. В случае дискретного четырехскоростного газа абсолютно максвелловское распределение задается постоянными решениями уравнения $I(f) \equiv f_{3} f_{4}-$ $f_{1} f_{2}=0$. Итак, получаем следующие условия:

$$
\begin{gathered}
\left.f_{j}(r, \varphi)\right|_{r \rightarrow \infty}=f_{j}^{\infty}=\text { const, } \quad j=1, \ldots, 4, \\
f_{1}^{\infty} f_{2}^{\infty}=f_{3}^{\infty} f_{4}^{\infty} .
\end{gathered}
$$

Также средняя скорость газа на бесконечности равна нулю:

$$
f_{1}^{\infty}-f_{2}^{\infty}=0, \quad f_{3}^{\infty}-f_{4}^{\infty}=0 .
$$

Определим полную плотность газа на бесконечности равенством

$$
\rho^{\infty}=\sum_{j=1}^{4} f_{j}^{\infty}=4
$$


Из граничных условий на бесконечности, т. е. из (12)-(14), следует, что $f_{j}^{\infty}=1$, $j=1, \ldots, 4$. Тогда решения краевой задачи (11)-(14) для системы Бродуэлла (1) задаются равенствами (4) при $C=0, K=1$. В полярных координатах эти решения имеют следующий вид:

$$
f_{1,2}(r, \varphi)=1 \mp \frac{\cos \varphi}{r}, \quad f_{3,4}(r, \varphi)=1 \mp \frac{\sin \varphi}{r} .
$$

Рассмотрим компоненты вектора импульса газа

$$
\rho v_{x}=f_{1}-f_{2}=-2 \frac{\cos \varphi}{r}, \quad \rho v_{y}=f_{3}-f_{4}=-2 \frac{\sin \varphi}{r} .
$$

Теперь, переходя к полярным координатам, для вектора импульса газа получаем выражение

$$
\rho \vec{v}=\rho v_{r} \vec{e}_{r}+\rho v_{\varphi} \vec{e}_{\varphi}=-\frac{2}{r} \vec{e}_{r}
$$

где $\vec{e}_{r}, \vec{e}_{\varphi}$ - орты полярной системы координат. Таким образом, поле скоростей газа аксиально-симметрично и направлено в сторону полости, т. е. полость поглощает газ из внешнего пространства.

Так как явный вид функции распределения задачи известен (см. (15)), то можно точно найти гидродинамические моменты любого порядка. Рассмотрим тензор напряжений. Известно, что он может быть выражен через функцию распределения следующим образом [14]:

$$
P_{i j}=\int c_{i} c_{j} f(\xi) d^{2} \xi, \quad i, j=1,2,
$$

где $\xi$ - молекулярная скорость, а $c_{k}=\xi_{k}-v_{k}, k=1,2$. Для модели Бродуэлла (1) имеется всего четыре возможные скорости движения частиц, поэтому интеграл в последней формуле заменяется суммой. Например, недиагональный элемент тензора напряжений имеет вид

$$
\begin{aligned}
P_{12}=(1 & \left.-v_{x}\right)\left(-v_{y}\right) f_{1}+\left(-1-v_{x}\right)\left(-v_{y}\right) f_{2}+ \\
& +\left(-v_{x}\right)\left(1-v_{y}\right) f_{3}+\left(-v_{x}\right)\left(-1-v_{y}\right) f_{4}=-\frac{x y}{\left(x^{2}+y^{2}\right)^{2}}
\end{aligned}
$$

Нетрудно видеть, что тензор $P_{12}$ также выражается через первые производные от средних скоростей:

$$
P_{12}=-\mu\left(\frac{\partial v_{x}}{\partial y}+\frac{\partial v_{y}}{\partial x}\right)
$$

где безразмерный коэффициент вязкости $\mu=1 / 2$. Аналогичным образом для диагональных элементов тензора напряжений получаем

$$
p_{11}=P_{11}-p=P_{11}-\frac{1}{2}\left(P_{11}+P_{22}\right)=\frac{1}{2}\left(P_{11}-P_{22}\right)=\frac{x^{2}-y^{2}}{2\left(x^{2}+y^{2}\right)^{2}}=-2 \mu \frac{\partial v_{x}}{\partial x},
$$

где коэффициент вязкости $\mu=1 / 2$. Итак, тензор напряжений пропорционален тензору скоростей деформаций. Такие же соотношения наблюдаются между этими тензорами в гидродинамике. 
Теперь следует установить, какая связь имеется между вектором потока тепла и градиентом температуры. Имеем для температуры $T$ следующее выражение:

$$
T=\frac{1}{2 \rho}\left(P_{11}+P_{22}\right)=\frac{1}{2}-\frac{1}{8\left(x^{2}+y^{2}\right)} .
$$

Для потоков тепла выполняются равенства [14]

$$
q_{i}=\int c_{i}\left(c_{1}^{2}+c_{2}^{2}\right) f(\xi) d \xi, \quad i=1,2 .
$$

В силу дискретности возможных скоростей частиц интеграл заменяется суммой. В итоге получаем

$$
q_{1}=-\rho v_{x}-\lambda \frac{\partial T}{\partial x}, \quad q_{2}=-\rho v_{y}-\lambda \frac{\partial T}{\partial y},
$$

где безразмерный коэффициент теплопроводности $\lambda=4$. Из соотношений (17) следует, что поток тепла хотя и пропорционален градиенту температуры, но еще зависит от импульса газа. Подобного не наблюдается в гидродинамике. Более того, поток тепла направлен во внешнее пространство, хотя из равенства (16) следует, что края полости, находящейся в начале координат, поддерживаются при температуре более низкой, чем температура в пространстве. Для поддержания такого состояния требуется постоянно отводить тепло от полости во внешнее пространство - этому как раз и соответствуют первые слагаемые в правых частях формул (17). Таким образом, для данной задачи непригодна теория сплошной среды, так как система сильно неравновесна. Заметим, что в сильно неравновесных случаях, например в некоторых задачах о неоднородной пространственной релаксации, знаки градиента температуры и теплового потока могут даже совпадать [15] (когда релаксация происходит в зоне длины свободного пробега).

\section{5. ЗАКЛЮЧЕНИЕ}

В настоящей работе были построены три новых класса решений четырехскоростной дискретной модели Бродуэлла, отвечающие несжимаемому и сжимаемому газу. Решения имеют автомодельный вид и выражаются через элементарные функции. Для всех решений могут быть поставлены задачи об испарении и конденсации газа в прямоугольной области (9), (10). Решения (15) также удовлетворяют задаче об испарении и конденсации газа на границе круга и внешнего пространства (11)-(14). Показано, что система сильно неравновесна и для нее неприменимы уравнения сплошной среды. K настоящему моменту неизвестно, какие стационарные задачи системы Бродуэлла устойчивы или неустойчивы. Как уже отмечалось выше, система Карлемана допускает хаотическую динамику [10], это позволяет сделать предположение, что для более сложных дискретных кинетических моделей могут наблюдаться хаотические режимы. Таким образом, аналитическое исследование и численное моделирование нестационарных возмущений стационарных задач системы Бродуэлла представляют значительный интерес для будущих исследований.

Благодарности. Автор выражает благодарность В.В. Аристову за полезные обсуждения. 


\section{Список литературы}

[1] J. Broadwell, J. Fluid Mech., 19 (1964), 401-414.

[2] H. Cornille, J. Phys. A, 20:16 (1987), L1063-L1067.

[3] H. Cornille, J. Statist. Phys., 52:3-4 (1988), 897-949.

[4] A. V. Bobylev, G. Spiga, J. Phys. A, 27:22 (1994), 7451-7459.

[5] A. V. Bobylev, G. Caraffini, G. Spiga, Eur. J. Mech. B/Fluids, 19:2 (2000), 303-315.

[6] A. V. Bobylev, Math. Meth. Appl. Sci., 19 (1996), 825-845.

[7] H. Cabannes, Eur. J. Mech. B/Fluids, 16:1 (1997), 1-15.

[8] О. Линдблом, Н. Эйлер, ТМФ, 131:2 (2002), 179-193.

[9] A. Bobylev, G. Toscani, Contin. Mech. Thermodyn., 8:5 (1996), 257-274.

[10] V. Aristov, O. Ilyin, Phys. Lett. A, 374:43 (2010), 4381-4384.

[11] В. Аристов, Ж. вычисл. матем. и матем. физ., 44:6 (2004), 1127-1140.

[12] F. Golse, Commun. Partial Differ. Equations, 12:3 (1987), 315-326.

[13] C. Cercignani, R. Illner, M. Shinbrot, Commun. Math. Phys., 114:4 (1988), 687-698.

[14] М. Коган, Динамика разрежсенного газа, Наука, М., 1967.

[15] V. Aristov, Phys. Lett. A, 250:4-6 (1998), 354-359.

Поступила в редакцию 10.04.2011 